\title{
Socioeconomic technologies - development trends in the age of digitalization
}

\author{
L.A. Reingold ${ }^{1}$, A.V. Solovyev ${ }^{2}$, E.A. Reingold ${ }^{3}$ \\ leonidrein@gmail.com |soloviev@isa.ru|1_r@mail.ru \\ ${ }^{1}$ LLC DIAVER Moscow, Russia \\ ${ }^{2}$ Federal Research Center "Computer Science and Control” of Russian Academy of Sciences, Moscow, Russia \\ ${ }^{3}$ LLC MCD PARTNERS Saint Petersburg, Russia
}

\begin{abstract}
Dramatical changes in society's infrastructure require new conceptual approaches towards the research of socioeconomic phenomena. Nowadays, environment surrounding people experience qualitative changes; it has become possible to use full information on the history of objects. It is essential to develop a conceptual framework enabling to thoroughly describe the interaction of an individual and a socioeconomic environment that is changed by digital technologies. In this article, it is suggested to use the concept of socioeconomic technology in the context of exploration of digitalization processes. These include the Internet of things, big data processing, widespread implementation of personal tools to process and share information etc. The concept of socioeconomic technology allows tying up new technologies with the needs of an individual and society as well as with phenomena emerging in socioeconomic infrastructure. The suggested approach may be used at the development and implementation of the Internet of things, of global communication infrastructure, of technologies for processing and storage of data, and for the research of other aspects of society's digitalization. Today it is possible to research different directions for the development of consumption sphere and technologies taking into account the emergence of virtual properties of objects. Virtual properties result for example in multiple ways to implement things, in the possibility to store and use the full history of major objects surrounding an individual. Conceptual framework suggested in this article allows holistically addressing the issues of commercial efficiency and socioeconomic management in the age of digitalization.

Keywords: digitalization, socioeconomic technology, the Internet of things, virtual properties of an object, big data, digital technologies.
\end{abstract}

\section{Introduction}

Digitalization and a widespread implementation of digital technologies result in the fundamental changes of the socioeconomic infrastructure of modern society. Providing this, there is a high demand for the tools allowing studying and assessing the consequences of the implementation of digital technologies. It is required to study thoroughly the needs specific to the current socioeconomic system emerging due to the development of new methods and technologies to satisfy them.

The use of digital technologies provokes virtualization and complication of socioeconomic mechanisms on different levels of society. Things obtain new implicit properties due to the availability of imbedded data processing tools, management and communication instruments. These enable new ways to satisfy individual's needs. These phenomena must be taken into account at the development of digital and social infrastructures of the modern society.

Nowadays, new consumer goods are emerging quite fast, existing goods are modified due to the application of digital technologies for the design and manufacture; interaction with environment is being automated. The emergence of new properties in objects is a source of new challenges for both society in general and an individual personally. For example, the implementation of new infrastructure is complicated by privacy issues, personal and financial security challenges etc.

There are different approaches towards the development of digital infrastructure so that it is more user-friendly. One of the approaches enabling faster adaptation to new conditions is the implementation of new features by nature-like methods, i.e. by familiar and understandable methods [1]. The use of artificial intelligence technologies, robotic engineering that considers the peculiarities of human brain can make digital infrastructure even more user-friendly [2]. It is needed to analyze conceptual issues, socioeconomic consequences arising out of the implementation of digital technologies.

Before digitalization, the methods of manufacturing and consumption of objects surrounding individuals did not change much. At present, the integration of virtual properties into objects, the development of information processing and communication systems made the structure of social interaction changeable. There is a need for conceptual and technological solutions covering the whole life cycle of objects surrounding individuals and allowing detecting and addressing challenges arising on the way. For example, there are new issues in the sphere of state administration and legal regulation. The necessity to address these issues is recognized by the society, which is supported by management principles and statutory framework in use, including in Russia [3, 4].

It is crucial to conceptualize the changes at the mass implementation of end-to-end digital technologies: big data, virtual and augmented reality technologies, distributed ledger systems etc. [5]. Digital ecosystems that connect people, things and virtual objects for co-use of digital platforms are being developed [6]. The implementation of such systems considerably change society's major life spheres - manufacture, consumption, management systems on all levels. The changes affect all spheres of life - career, lifestyle, leisure, communication with other individuals etc.

Digital technologies generate huge data volumes, enable its long-term storage and real-time usage. Interests of all involved users should be considered when using this data; all encountered complications should be dealt with proactively.

The majority of new methods to satisfy the needs appear due to the emergence of new features enabled by the implementation of digital technologies. To address arising scientific and practical tasks is it needed to take 
into consideration variability and multilevel structure of changes in manufacturing and consumption patterns occurring in the society in general and in the life of every individual. Tools allowing exploring emerging opportunities and defining their usage terms and applicability limits are in demand. Some approaches towards development of such tools are described herein.

\section{Socioeconomic technology}

Technologies are intended for the satisfaction of needs of an individual, a social group or society in general. The needs are defined by human body characteristics, social group activities, society's requirements, manufacturing capabilities etc. We can consume natural and artificial objects. The integration of digital technologies enables to satisfy needs on a new qualitative level, by methods that are more convenient.

Nowadays, the consumption process is defined in the context of manufacturing capability and existing demand - any opportunity to meet the demand is fulfilled. However, in the age of digitalization this approach is not enough. A comprehensive analysis of consequences after the implementation of digital technologies should be done because the changes it provokes are variable, deep and controversial.

Using terminology of databases developers, the correlation between technologies and needs can be described as many-to-many - one technology can be used to satisfy many needs, while one need can be satisfied by several technologies [7]. To describe this correlation we suggest the concept of socioeconomic technology (hereinafter SET) [8]. The correlation between technologies and needs is not conceptually developed and thought over.

The SET concept is not well-defined and is usually used in economic, sociological and philosophical spheres. However, this concept can be effectively applied to the research of digital transformation processes.

Under SET we mean the satisfaction of a certain need with the use of a certain technology. For example, people always wanted to exchange messages. In the course of time, more advanced and convenient technologies were used to meet this need: from birch-bark manuscripts and letters mailed by postal service to emails and SMS and other messengers. Appeal of this type of SET to consumers improves when digital technologies are applied. Quality parameters for this type of SET include message transfer rate, price of delivery, size and length of the messages, possibility to communicate in dialog, group communication etc.

It could take months to deliver a letter while in digital environment information travels instantly. Messages can include audio and video data. Thus, SET is always changing according to the users' needs and requirements.

Technologies implemented in the course of digitalization do not always substitute previously used technologies. Quite often, they are used to modify existing ways to meet the needs. SET of the same application that emerged in different time and that are based on different technologies can coexist for a long time and serve a particular niche of users satisfying their needs. As far as information is concerned, digital technologies allow providing a new quality level of situational awareness of a user. Traditional mail successfully coexist with digital communication technologies although it is gradually forced out by other means of communication because the use of technical means is not always supported by regulatory procedures and instruments.

SET is evolving technologically - technologies and processes behind them, regulatory support is changing. New SET allow meeting new needs and requirements. The progress of modern society is largely the result of advancement of existing and development of new SET enabling the evolution of manufacturing and consumption processes.

Fig. 1 illustrates the concept of socioeconomic technology. In the left part of the scheme, there are technologies enabling technological means for the implementation of SET. In the right part of the scheme, there are needs and requirements of a certain type of individuals. At the intersection of technologies and needs there are SET. New SET are emerging as a combination of available technologies and existing needs. A SET should be sought-after and its implementation should be possible with the use of available technologies. Efforts to satisfy the needs are based on a certain business model that guarantees profitability if they are designed for commercial use. Besides, SET can serve as a tool of socioeconomic management supporting, for example, the implementation of regulatory documentation.

SET system that uses digital infrastructure operates automatically. Human involvement into SET tends to decrease due to the automatization of business processes that in its turn is provoked by algorithmization, implementation of artificial intelligence and robotization. The architecture of enterprises in digital environment changes, which is reflected in corresponding standards [9].

The use of SET concept allows exploring the system to satisfy the needs in connection with new technologies, to examine consumption mechanisms in their entirety.

In literature, the expression "socioeconomic" characterizes a certain technology either in social or economic context. The concept suggested in this article is more defined and allows applying a new method to explore ongoing changes.

A new type of objects - SET - is introduced; it links the need with the technology used to meet it. This link is named, it can have identification and other attributes, it can be researched along with other similar objects. Such approach enables a thorough detailed analyses and process modeling of digital transformation. It is possible to develop methods to build new SET or analyze consumer behavior in new conditions.

Consumption process is usually divided in time. New technologies are not implemented instantly - their development, implementation and regulatory support of their application take time. Implementation of new SET compared to existing ones as a rule requires investments and a certain additional knowledge. Providing this, the usage of new SET is low at the beginning but it grows with the course of time and availability. The use of digital technologies increases attainable level of consumption the speed of needs satisfaction is growing, participation of 
other individuals to meet the need is not required more often than not, the costs are decreasing etc. Thus, the development of modern society is substantially defined by digital technologies.

SET can be analyzed on different levels of generalization. Both technologies and needs can be demonstrated in the form of multilevel classification. Providing this, SET is a derivative classification whose every element is defined by corresponding elements from technologies and needs classifications.

In the example with messages above, two classification options are possible - on the one hand, a classification of messaging technologies, on the other, a classification of needs for sharing information. A combination of applied technologies and existing needs that does not go beyond the limits of common sense forms an admissible set of SET.

It is known that classifications can be monohierarchical and polyhierarchical [10, p.17]. Polyhierarchical classifications permit multiple inheritance, i.e. they allow demonstrating that SET may be based on several technologies and several needs from different levels of their classification. This is schematically demonstrated on figure 1 . The use of this approach allows presenting a formalized SET description on different levels of generalization. Classifications of needs and technologies applied for the certain object domain form the basis for such SET classification.

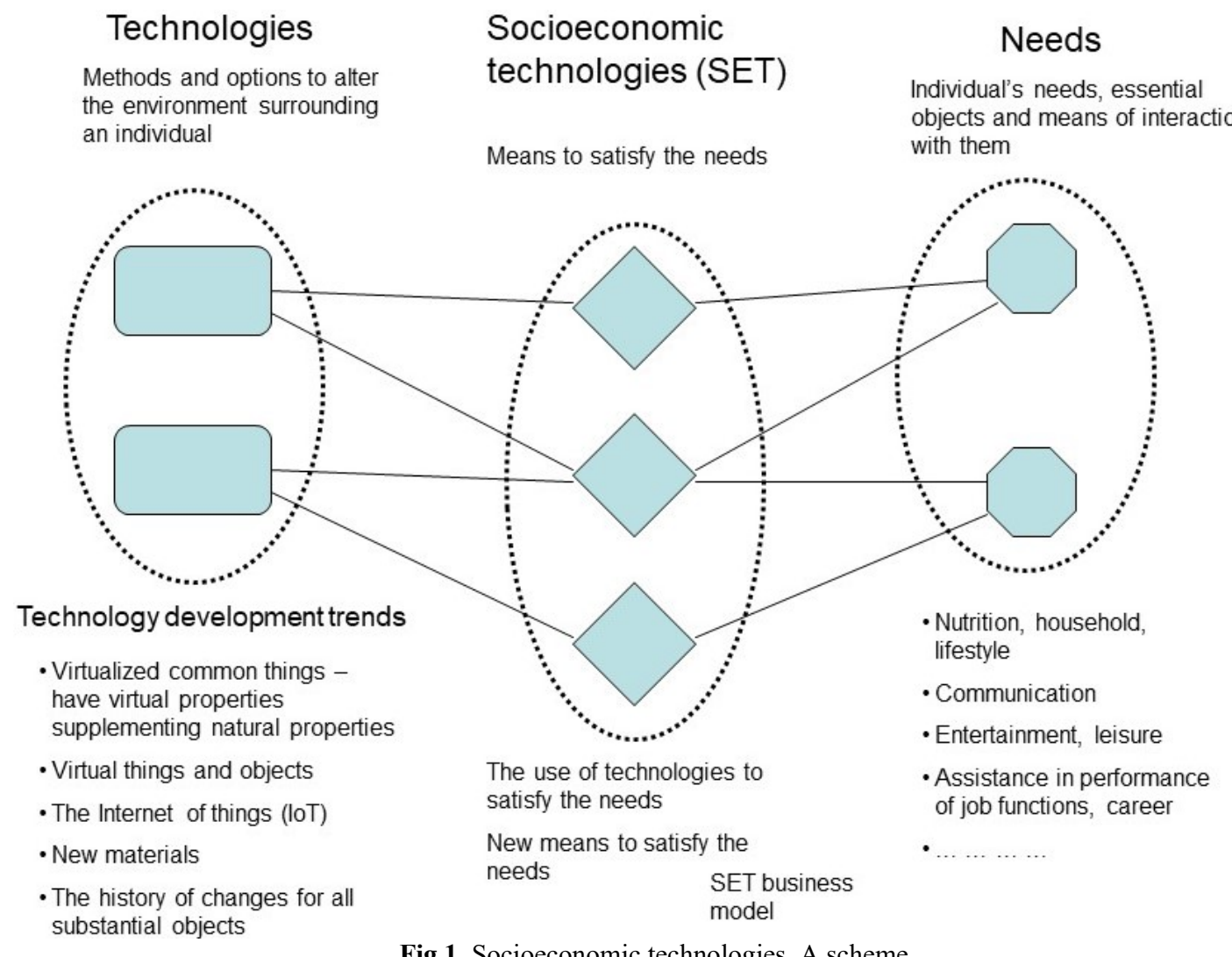

\section{Socioeconomic digital technologies}

The implementation of digital technologies increases the number of SET variants - new virtual or virtualized (having explicit virtual properties) objects emerge; the infrastructure of society is interconnected by a general communication system. There were no previous analogues to some of the technologies, their capabilities result in the emergence of new needs.

The examples of such previously non-existent technologies are geolocation and automatic objects recognition. People, vehicles and other objects of natural and created environment are the SET objects based on these technologies. With the use of these SET the scope of activities and object's location can be stored and later used to meet the needs of individuals or improve the performance of socioeconomic system in general.
Thus, the emergence of navigation services for vehicles on the one hand allows saving time and resources of a certain individual, on the other hand it helps to improve the performance of the transportation system in general. Such technologies make it possible to meet the existing needs on a different level and at the same time they are the basis for the development of new needs and requirements and their further addressing with new SET. A digital ecosystem is formed around such technologies; it allows developing SET with new functional capabilities using emergent features of such a system.

The examples of digital technologies that form the basis for the emergence of new methods to satisfy communication needs include new generation communication technologies such as $5 \mathrm{G}$ for establishing connectivity between devices on large territories (a city, a state, the planet in general) [11]; WiFi 6 enabling data exchange within buildings and confined spaces [12]; Bluetooth 5.1 enabling to connect devices of an individual 
taking into account their location [13]. SET created on the basis of these technologies will be able to meet individuals' communication needs and support the performance of the emerging infrastructure of the Internet of things in future.

A modern individual expects that the performance of digital objects will be programmable and intellectual. The needs of individuals may be classified by different parameters. For example, we can define the following basic groups of needs - nutrition, household, and lifestyle; communication between themselves and with surrounding automated devices; entertainment and leisure; assistance in performing job functions etc.

SET can be different even when the same technologies are used to satisfy the same needs because SET performance can be implemented differently from technological and managerial point of view. For example, different business models or conceptual solutions may be used at the different levels of applied technological stack. Data storage may be implemented using different approaches towards data storage, different data base management systems, different data access methods etc.

Let us discuss the structure of SET considering peculiarities of digital technologies used in it. SET consists of the following elements - technological stack, the description of a need SET meets, parameters specifying the SET in general. These elements include:

- Specifics and functional features of the used technologies in general and of the digital technologies stack applied at the implementation of SET.

- Description of a need satisfied by SET and SET users' characteristics.

- Parameters defining SET in general. These are the parameters describing the main functional, technical and economic features of SET as a universal system and distinctive features of SET.

Development and implementation of SET require resources, namely - design of digital devices, analyses of consumers' needs, implementation of digital infrastructure. Training of users and marketing of new SET is also needed. Development and operation of a socially valuable SET is covered from the state's budget, while the use of a commercial SET for the provision of goods and services is paid for. In many cases, the result of digital SET application is information. Thus, SET supplier often gets the payment for the use of SET not in monetary form, but in the form of access to the information about service users; besides, the suppler can show advertising messages to users.

Let us define certain specifics of SET application that are important for the correct usage of this concept:

- As a rule, SET can be considered on different levels of generalization. For example, messaging technology can be considered in general or we can discuss certain messaging technologies - a letter, an email, an SMS etc.

- A need can be satisfied by different SET, there are always several variants, and individuals can choose the means to meet their need. For example, one can send a letter over a regular mail or electronically - by email, a social network or any messenger service.
- A need does not depend on the existence of a SET that can meet it. A need can be first conceived and later the technology and SET to satisfy it will be suggested. For instance, the desire to fly emerged by far earlier than the possibility to do so.

Understanding of the need allows formulating the requirements towards SET and finding the methods to satisfy it among available technologies. The research of needs as one of the main conditions for the development of SET is growing considerably in the age of digitalization due to the growing flexibility of infrastructure. The reason for the that is that it is much easier to implement digital properties with the use of digital technologies built into things than their development by traditional "mechanical" or "electrical" methods.

Flexibility of functionality specific to things, emergence of elements of intelligent behavior and mutual communication result in additional challenges. Legallybinding agreements that are automatically generated, smart contracts regulating concerted behavior of such things should be developed [14].

Providing the above, it should be noted that the planing of needs is more important than their spontaneous development. Traditional marketing may provoke system problems. One of the examples of problematic spontaneous development of digital technologies is the use of new communication features of social networks in order to support illegal activities.

The use of SET concept to explain digitalization processes allows implementing a new approach towards detailed description of consumption system in the age of digitalization. It allows developing the means of automation for the exploration of consumption system and avoid a number of problems in the course of implementation of new technologies. Let us discuss specifics and challenges related to the application in SET of some popular digital technologies, namely the Internet of things, big data and individual communication devices of citizens.

\section{The specifics of socioeconomic technologies usage in digital environment}

Digital technologies have certain specific features people were unaware of previously. These include data processing methods in digital environment and approaches towards addressing challenges related to them.

The important features of digital infrastructure are the ability to store and process data, informative interaction of infrastructure's elements, a possibility to store the history of previous parameters for all significant objects. At the same time, these features cause problems. For example, there are certain issues when data is stored for a long time, when it is collapsed or when it is needed to guarantee its integrity because information has become a crucial resource for the functioning of modern society on all levels [15-19]. To avoid problems at the storage and processing of data the following aspects should be taken into account:

- Data format compatibility should be implemented on both technical and semantic levels.

- Data immutability is guaranteed. This applies to the preservation of semantics of regulatory and reference 
information in all collaborating systems and to the provision of immutability of values for the stored properties of objects.

- The information should be correctly collapsed when there is a lot of raw data from different sensors, IoT and communication devices.

- Authorized use of information should be guaranteed, including data access restrictions, control over the functionality of data enrichment by tying up data about objects generated from different sources with the use of big data technologies. Data enrichment control should be permanent because otherwise in the course of time once anonymous data can become personalized when new data is added into the system or new algorithms are applied to its processing.

- The change of restriction rules on the use of information when data is circulating for a long time should be eliminated. There are known cases when license agreements and other labilities were changed by the owners of automated systems without due notification of users.

Both the aspects listed above and other characteristics of digital technologies should be sustained by the digital infrastructure and regulatory system of the state.

\section{Conclusion}

In the age of digitalization, it has become essential to design methods enabling the elaboration of digitalization processes, the formalization and modeling of interaction between the needs and digital technologies. The SET concept makes it possible to study in detail and analyze the correlation between new technologies and needs they satisfy. The conducted research allows us to draw the following conclusions:

Digital technologies significantly and quickly change the structure and methods for the satisfaction of needs. Thus, it is important to address the technological aspect of SET and the needs in complex.

SET in digital environment obtain new properties: variability, virtuality, capability to use global communications etc. Individuals and society in general have conceptually new functional methods to ensure situational awareness and improve management, including identification and geolocation of all significant objects, storage of information about previous behavior and the use of this data in real time. These phenomena should be explored along with the options to satisfy the needs on a new level.

The use of digital technologies requires a conscientious approach towards the formation of needs. The use of SET concept allows describing formally the system of needs, assessing holistically the changes in technological capabilities and forecasting socioeconomic changes in the society.

The suggested conceptual approach will enable researching the specifics of manufacturing and consumption in the age if digitalization. The application of this approach may be automated and contribute to the addressing of issues of technological development and socioeconomic management in new conditions.

\section{Acknowledgements}

This article is prepared with a partial financial support of RFBR under the scientific project № 18-29-03070.

\section{References}

[1] Reingold L.A., Reingold E.A., Kopylov G.I. Information technology and nature similarity in the development of the infrastructure of society. Journal of Applied Informatics, Vol. 14, No. 5 (83), 2019. P. 53-73. DOI: 10.24411 / 1993-8314-2019-10036.

[2] Shumsky, S. A. Machine intelligence: essays on the theory of machine learning and artificial intelligence. Moscow Institute of Physics and Technology (National Research University). - Moscow: RIOR, 2019. - 339 p. ISBN 978-5-369-02011-1

[3] Program "Digital Economy of the Russian Federation". Approved by Order of the Government of July 28, 2017 No. 1632

[4] Order of the Government of the Russian Federation of March 23, 2018 N 482-r On the action plan ("road map") to improve legislation and remove administrative barriers in order to ensure the implementation of the National Technological Initiative in the direction of "Technet" (advanced production technologies). Access mode: http://assets.fea.ru/uploads/fea/nti/docs/rasporyazhen ie-dk-23032018-482.pdf (accessed 22.03.2020).

[5] Digital technology. Ministry of Digital Development, Telecommunications and Mass Media of the Russian Federation. Available https://digital.gov.ru/ru/activity/directions/878/ (accessed 22.03.2020).

[6] Akatkin Yu.M., Karpov O.E., Konyavsky V.A., Yasinovskaya E.D. Digital Economy: The Conceptual Architecture of the Digital Industry Ecosystem. Business Informatics. 2017. No. 4 (42). P. 17-28.

[7] What is Entity Relationship Diagram (ERD)? Available at: https://www.visualparadigm.com/guide/data-modeling/what-is-entityrelationship-diagram/ (accessed 22.03.2020).

[8] Reingold L.A., Slavin O.A. Socio-economic technologies as a generalization of trends in socioeconomic development // Proceedings of the Institute for System Analysis of the Russian Academy of Sciences. Intelligent information technology. Applied aspects. M.: 2005. P. 40-55.

[9] ISO 15704:2019 Enterprise modelling and architecture - Requirements for enterprisereferencing architectures and methodologies Available https://www.iso.org/standard/71890.html (accessed 22.03.2020).

[10] FIBO conceptual methodology, Further exploration of conceptual Modeling Principles FDTF Day 2, 17 Sept 2014. p. 17 Available at: http://www.hypercube.co.uk/edmcouncil/docs/fibobco-conceptual-modeling v0.2.pptx (accessed 22.03.2020).

[11] DeLooper Christian What is 5G? 5G's arrival is transforming tech. Here's everything you need to know to keep up. 24.01.2020. Available at: 
https://www.digitaltrends.com/mobile/what-is-5g/

(accessed 22.03.2020).

[12] Wi-Fi 6 in the enterprise: We're in a new wireless era. Available at: https://www.wi-fi.org/beacon/davechen/wi-fi-6-in-the-enterprise-we-re-in-a-newwireless-era (accessed 22.03.2020).

[13] Hoffman Chris. Bluetooth 5.1: What's New and Why It Matters. 31.12.2019 Available at: https://www.howtogeek.com/403606/bluetooth-5.1whats-new-and-why-it-matters/ (accessed 22.03.2020).

[14] Grin O.S., Grin E.S., Solovyov A.V. The Legal Design of the Smart Contract: The Legal Nature and Scope of Application. Lex Russica. 2019;(8):51-62 [Grin' O.S., Grin' Ye.S., Solov'yev A.V. Pravovaya konstruktsiya smart-kontrakta: yuridicheskaya priroda i sfera primeneniya. Lex russica (Russkiy zakon). 2019;(8):51-62.] .

[15] Bakanova, N.B., Soloviev, A.V. Problems of longterm keeping of big data // Information Technology and Computing Systems, No. 2, M.: 2019. P.44-53. DOI 10.14357/2071863219020.

[16] Solovyev, A.V, Danilenko, A.Yu., Akimova, G.P., Bogdanov, D.S., Pashkin, M.A., Pashkina, E.V., Podrabinovich, A.A., Tumanova I.V. Mathematical models for assessing the integrity of digital data transfer in a digital economy // Proceedings of the Institute for System Analysis of the Russian Academy of Sciences (ISA RAS), Vol. 69, Issue 2, M.: 2019. P.65-74. DOI: 10.14357/20790279190207.

[17] Zakharova A.A., Podvesovskii A.G., Shklyar A.V. Visual and cognitive interpretation of heterogeneous data (2019), Int. Arch. Photogramm. Remote Sens. Spatial Inf. Sci., XLII-2/W12, pp. 243-247. doi: 10.5194/isprs-archives-XLII-2-W12-243-2019 B

[18] Khakimova A.Kh., Zolotarev O.V., Berberova M.A. Visualization of bibliometric networks of scientific publications on the study of the human factor in the operation of nuclear power plants based on the bibliographic database Dimensions. Scientific Visualization, 2020, volume 12, number 2, pages 127 - 138, DOI: 10.26583/sv.12.2.10, E-ISSN:2079-3537.

[19] Zakharova, A.A., Nebaba, S.G., Zavyalov D.A. Algorithms and Software Increasing the Efficiency of Processing Multidimensional Heterogeneous Data (2019) Programming and Computer Software, 45 (4), pp. 196-201. doi: 10.1134/S0361768819040108.

\section{About the authors}

Reingold Leonid A., consultant, LLC DIAVER, Candidate of Technical Sciences. E-mail: leonidrein@gmail.com.

Solovyev Alexander V., Chief Researcher, Department 94 ISA FRC CSC RAS. Doctor of Technical Sciences. E-mail: soloviev@isa.ru.

Reingold Elena A., lead consultant, LLC MCD PARTNERS, $\mathrm{PhD}$ in Economics, docent. E-mail: 1_r@mail.ru. 